\title{
Chemical Conversion of TNT: Production of 2,4,6-Trinitrobenzoic Acid
}

\section{August 2000}

\author{
A. A. Asrat'ev \\ V. A. Marchukov \\ V. G. Suschev \\ A. V. Aleksandrov \\ V. V. Semenov \\ A. C. Buchanan III \\ A. A. Gakh
}




\section{DISCLAIMER}

This report was prepared as an account of work sponsored by an agency of the United States Government. Neither the United States Government nor any agency thereof, nor any of their employees, makes any warranty, express or implied, or assumes any legal liability or responsibility for the accuracy, completeness, or usefulness of any information, apparatus, product, or process disclosed, or represents that its use would not infringe upon privately owned rights. Reference herein to any specific commercial product, process, or service by trade name, trademark, manufacturer, or favoring by the United States Government or any agency thereof. The views and opinions of authors expressed herein do not necessarily state or reflect those of the United States Government or any agency thereof. 


\title{
Chemical and Analytical Sciences Division
}

\section{CHEMICAL CONVERSION OF TNT: PRODUCTION OF 2,4,6-TRINITROBENZOIC ACID}

\author{
A. A. Asrat'ev* \\ V. A. Marchukov* \\ V. G. Suschev* \\ A. V. Aleksandrov* \\ V. V. Semenov ${ }^{\dagger}$ \\ A. C. Buchanan III
A. A. Gakh

\footnotetext{
"St. Petersburg Institute of Technology, SKTB “Technolog”

Sovetsky Pr., 33a, St. Petersburg, 193117, Russia

${ }^{\dagger}$ Zelinsky Institute of Organic Chemistry

Leninsky Pr., 47, Moscow, 117913, Russia
}

Date Published-August 2000

\author{
Prepared by the \\ OAK RIDGE NATIONAL LABORATORY \\ Oak Ridge, Tennessee 37831-6285 \\ managed by \\ UT-BATTELLE, LLC \\ for the \\ U.S. DEPARTMENT OF ENERGY \\ under contract DE-AC05-00OR22725
}




\section{CONTENTS}

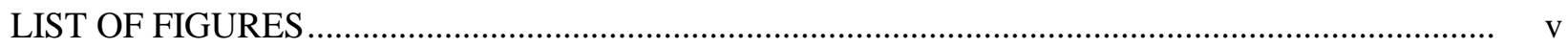

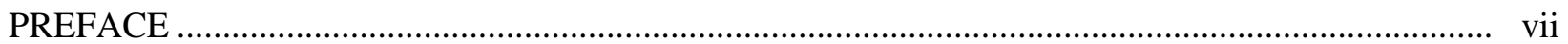



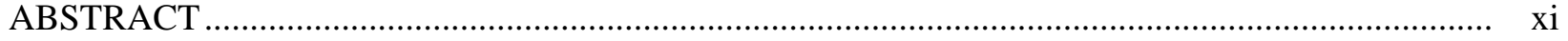

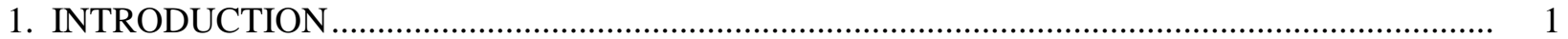

2. KINETICS OF TNT OXIDATION BY NITRIC ACID IN BATCH AUTOCLAVE ...................... 3

3. DESIGN AND TESTING THE METHOD FOR CONTINUOUS PRODUCTION OF TNBA IN A PILOT PLANT …....................................................................... 9

4. OXIDATION OF TNT TO TNBA ON AN INDUSTRIAL SCALE ................................................ 15

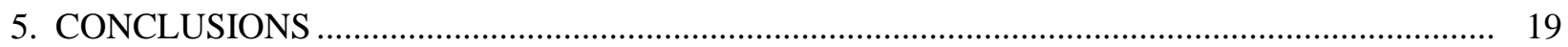






\section{LIST OF FIGURES}

Figure $\quad \underline{\text { Page }}$

2.1 Effect of temperature on TNT oxidation........................................................... 5

2.2 Effect of nitric acid concentration (C) on TNT oxidation kinetics............................ 6

$2.3 \quad$ TNT oxidation by nitric acid in autoclave. .............................................................. 7

$2.4 \quad 2,4,6-$ TNT oxidation by nitric acid. TNBA content in solid product (\% w/w) at different oxidation conditions. ........................................................................ 8

3.1 Principal scheme of pilot plant reaction for TNBA production................................. 10

S.2 Schematic diagram of oxidation reactor........................................................ 11

4.1 Flowchart illustrating TNBA production by TNT oxidation with nitric acid, including recycling of TNT .................................................................................. 16 


\section{PREFACE}

The Oak Ridge Site (ORS) is one of eleven Department of Energy (DOE) sites engaged in the Initiatives for Proliferation Prevention (IPP). The primary mission of IPP is to enhance the national security and nonproliferation objectives of the United States. These goals are achieved by providing meaningful, sustainable, non-weapons-related work for scientists and engineers in the Newly Independent States (NIS) that were formerly engaged in work concerning weapons of mass destruction. New businesses are established through the marriage of NIS scientists and technologies with U.S. companies, assisted by the oversight and technical expertise of personnel from the DOE sites, such as the ORS team of the Oak Ridge National Laboratory and/or the Y-12 Plant. The NIS countries involved in the IPP Program include Russia, Kazakhstan, and the Ukraine. The research reported here is a part of this ongoing effort. 


\section{ACKNOWLEDGMENTS}

This research was sponsored by the U.S. Department of Energy under the IPP program ("Chemical Conversion of TNT, ORS-TI-056-RU”). Oak Ridge National Laboratory is managed and operated by UT-Battelle, LLC, for the U.S. Department of Energy under contract DE-AC05-00OR22725. The Y-12 plant is managed and operated by Lockheed Martin Energy Systems Corporation for the U.S. Department of Energy under contract DE-AC05-84OR21400. 


\begin{abstract}
Oxidation of 2,4,6-trinitrotoluene (TNT) by aqueous nitric acid at high temperature and pressure gives 2,4,6-trinitrobenzoic acid (TNBA) and other valuable products, such as 1,3,5-trinitrobenzene (TNB). Optimization of the kinetics proved to be critical for the selective oxidation of the methyl group. High yield of a desired product can be obtained only under a narrow range of conditions. Thus, the best yield (70 to $75 \%$ ) of TNBA was achieved at a 35 to $45 \%$ conversion of TNT ( $80 \%$ nitric acid, $194^{\circ} \mathrm{C}, 20 \mathrm{~min}$ ), whereas the decarboxylation product (TNB) was the major component of the reaction mixture after a 50-min reaction. Subsequent separation of TNBA was achieved by selective extraction with aqueous bicarbonate. Practical technology development steps for a continuous mode of operation leading to the chief products are also discussed.
\end{abstract}

This technology can use commercial raw trotyl and trotyl from discharged ammunition as the starting material. The latter could be of particular importance for the conversion program aimed at the utilization of ammunition supplies. 


\section{INTRODUCTION}

2,4,6-Trinitrobenzoic acid (TNBA) can serve as a raw material for the synthesis of various products that are of considerable practical interest.[1-4] However, until now, there has been no satisfactory procedure for preparing TNBA, which can be a basis for industrial production of this compound. 2,4,6Trinitrotoluene (TNT) is a plentiful, available starting material for the production of TNBA by oxidation in strongly acidic media. Commonly used methods of oxidation by chromic acid or oleum-nitric acid mixtures result in the formation of large amounts of wastes that are difficult to utilize, and an end product that is difficult to purify.[5] Further, it is difficult to implement acid circulation and recycling in the experimental apparatus.

It is of interest that aqueous nitric acid can also be used as the oxidizing agent—readily regenerated within the technology cycle of the process using well-known industrially developed methods. In addition, the solutions can be concentrated by evaporation at reasonably low temperatures.

However, it has been shown that TNT can be oxidized by aqueous nitric acid solutions only at rather high temperatures under pressure.[6] It was found at the SKTB "TEKHNOLOG" that 1,3,5-trinitrobenzene (TNB) was formed as the single product in the treatment of TNT with nitric acid at a temperature higher than $220^{\circ} \mathrm{C}$ under pressure ( 8 to $10 \mathrm{MPa}$ ). On this basis, an industrial process for TNB production was developed at the SKTB "TEKHNOLOG." A commercial unit was designed, and a high degree of safety was provided in the use of thermal oxidation synthesis in nitric acid media under pressure. Moreover, it was found that commercial raw trotyl and trotyl from discharged ammunition can be used as the starting material. The latter is of particular importance in the context of the conversion program aimed at the utilization of ammunition supplies.

It was discovered in the course of previous studies on TNT oxidation that TNBA can be formed as an impurity in the reaction products at lower oxidation temperatures. However, this problem has received no significant attention because TNBA was not formed under the more severe conditions recommended for TNB production.

It may be suggested that TNBA can be obtained as the target product by oxidation of TNT under milder conditions. In this connection, a special study was conducted at the SKTB

"TEKHNOLOG." This report summarizes the results from this study.

It was found experimentally that TNBA is formed by the oxidation of TNT by nitric acid under mild conditions. Moreover, using kinetic factors, oxidation conditions that are optimal for the production of TNBA were determined.

The study of the kinetics of oxidation made it possible to determine (1) the ranges of optimum parameters and (2) the requirements imposed on the apparatus and process design for TNBA production.

Based on the results obtained, a pilot setup was built. The conditions of the synthesis were produced in this setup, and pilot batches of the product were prepared. A flowchart of the industrial manufacture was designed. 


\section{KINETICS OF TNT OXIDATION BY NITRIC ACID IN BATCH AUTOCLAVE}

Taking into account the experimental conditions (high temperatures and pressures, corrosive media, and explosive materials), the experiments were performed on a specialized autoclave bench modified for examining potentially hazardous thermal oxidation processes under pressure in aqueous nitric acid media.

This bench provides an opportunity to operate with autoclaves with remote control, to perform heating, thermostatting, and cooling of the autoclaves, to supply compressed gases, and to control and measure temperature and pressure parameters.

High-temperature, liquid-phase oxidation processes in aqueous nitric acid are autocatalytic reactions, and the product yield exhibits extremums. Because of this characteristic, the kinetic curves of TNT oxidation and the formation of the key reaction products were obtained and compared in this study.

The kinetic curves were constructed from the results of an analysis of reaction products obtained in a series of experiments performed under the same conditions but with different times of contact between the reactants at high temperatures.

The experiments were performed using special thin-walled-tube titanium autoclaves with a volume of 60 to $400 \mathrm{~mL}$. The reaction mixture in these reactors can be heated and cooled rapidly.

The usual experimental procedure is described below:

Starting reactants (TNT, aqueous nitric acid solution) were quantitatively loaded in the thinwalled-tube titanium autoclave. The autoclave was a cylindrical vessel with a cap with cartridges for a thermocouple and a joint for connecting a pressure gage and supplying a gas. The autoclave was hermetically sealed with a special valve and then placed in a thermostat with a liquid metal alloy (the Rose alloy, m.p. $94^{\circ} \mathrm{C}$ ), which was preheated to a specified temperature. The temperature and pressure changes in the autoclave were monitored in the course of heating and thermostatting of the autoclave. After the required exposure in the thermostat, the autoclave was removed and rapidly cooled with cold water. All operations involving heating, thermostatting, and cooling of the autoclave were controlled remotely.

After cooling, the autoclave was opened, and the liquid reaction mixture was diluted with water and quantitatively removed from the autoclave. Organic products were repeatedly extracted from the reaction mixtures using ethyl acetate.

The composition of the organic products was determined by high-performance liquid chromatography using (HPLC) an internal standard.

The HPLC parameters were as follows:

Column: length, $63 \mathrm{~mm}$; internal diameter, 2 $\mathrm{mm}$.

Sorbent: Silasorb $600 \mathrm{SpH}, 5.0 \mathrm{~mm}(\mathrm{La}$ Chema). Ultraviolet detector: detection wavelength, $254 \mathrm{~nm}$.

Eluent flow rate: $100 \mathrm{~mL} / \mathrm{min}$.

Samples were injected as solutions in ethyl acetate; the sample volume was $1 \mathrm{~mL}$.

The experiments were performed over the temperature range 160 to $220^{\circ} \mathrm{C}$ in aqueous nitric acid at concentrations of 50 to $90 \%$. A large excess of the oxidant was used in all of the experiments to decrease the effect of variations in the oxidant concentration over the course of the reaction. 
We found that TNBA was not formed in significant amounts when the oxidation was performed at temperatures over $200^{\circ} \mathrm{C}$ and at nitric acid concentrations lower than $60 \%$. However, at 180 to $190^{\circ} \mathrm{C}$ with nitric acid concentrations of 70 to $80 \%$ there is a time interval over which TNBA is not only present in the reaction mixture, but also is the major product of the reaction.

From the standpoint of the stability of the final products, the formation of TNB and the complete decomposition of organic compounds are the major competitive processes in the reaction. It is likely that these products are formed either as a result of secondary processes of TNBA transformation or as a result of direct oxidation of TNT. As a result, the time dependence of the TNBA yield exhibits an extremum, depending on the conversion, and the dependence of the yield on the process parameters is complicated.

Other oxidation products were not found in detectable amounts in the reaction mixture. Some quantities of picric acid were found under milder conditions (more dilute solutions, lower temperatures). However, under optimum conditions, this compound was absent in the reaction mixture and was ignored in the kinetic analysis.

Figures 2.1 and 2.2 show typical kinetic curves for TNT oxidation and TNBA and TNB buildup over the course of oxidation. The dashed line demonstrates the TNBA yield on the basis of converted TNT.

Note from the curves that the yield of TNBA at particular points in time can be higher than $80 \%$ on the basis of converted TNT. At the same time, the "practically important range" of operating parameters that correspond to high TNBA yields and reasonable rates of TNT conversion is rather narrow. Thus, a decrease in the nitric acid concentration below $70 \%$ or an increase in the process temperature above $200^{\circ} \mathrm{C}$ results in almost complete disappearance of TNBA in the reaction products. An increase in the conversion or the reaction time results in the change of the reaction pathways; thus, TNB becomes a sole product of the reaction. In all cases, the TNBA yield exhibits the extreme time dependence, leading us to conclude that optimization of the reactant contact time is essential.

In the region of high TNBA yields, the nonacid organic products (TNB and TNT) are always present in the reaction mixture. Thus, any method for preparing TNBA should simultaneously involve the separation of the target product. The ratio between the organic products in the reaction mixture can be varied within wide limits by varying the process parameters. Optimization criteria can be used, depending on the mode of further processing and technical limitations. For example, if unconverted TNT is recycled, the minimum TNB content in the reaction products, rather than a TNBA yield, is the crucial factor. It is likely that the TNBA yield will be a key factor if separated organic nonacid products are used as raw material for TNB production.

To reach the specified productivity, the reactant contact time should be the shortest possible, which could be accomplished by an increase in the temperature. However, under these conditions, the operating pressure in the apparatus and the extreme character of the interval of the optimal residence time increased. The maintenance of stable parameters is more important. For this purpose, the region of the optimum contact time of the reactants should be broadened. A decrease in the temperature, removal of dissolved nitrogen oxides, and an increase in the pressure are favorable factors for the broadening of this range. 
TNT oxidation by $80 \%$ nitric acid at $184^{\circ} \mathrm{C}, \mathrm{M}=41.4$

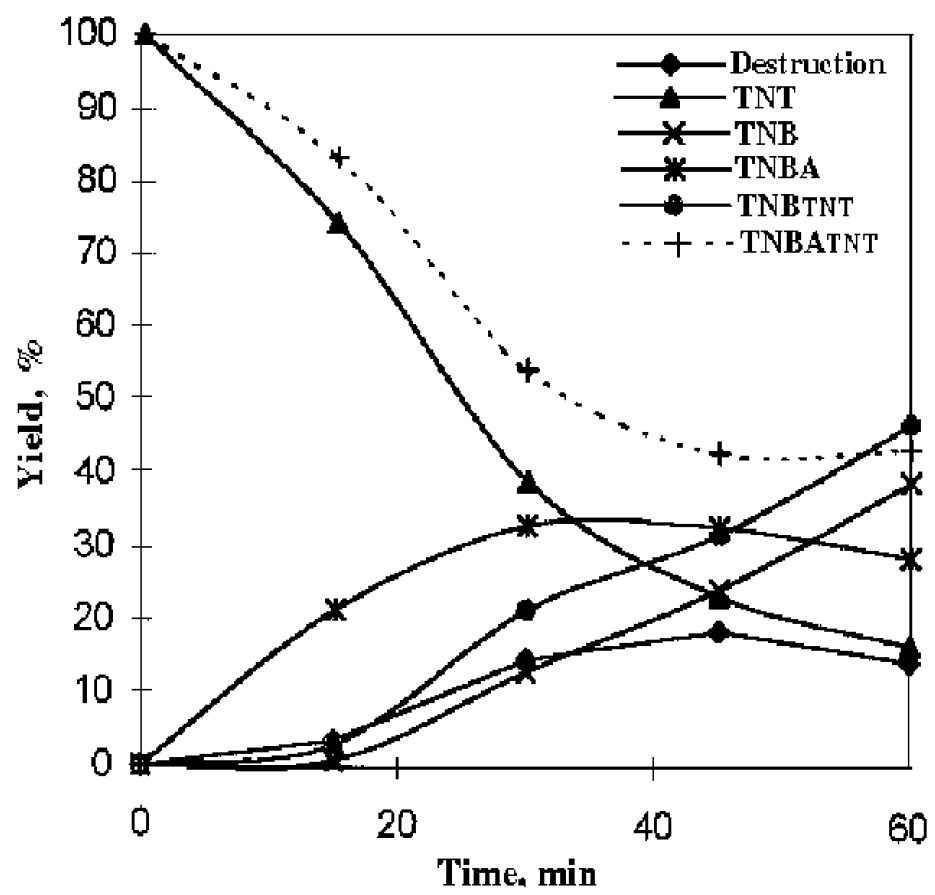

TNT oxidation by $80 \%$ nitric acid at $194^{\circ} \mathrm{C}, \mathrm{M}=41.4$

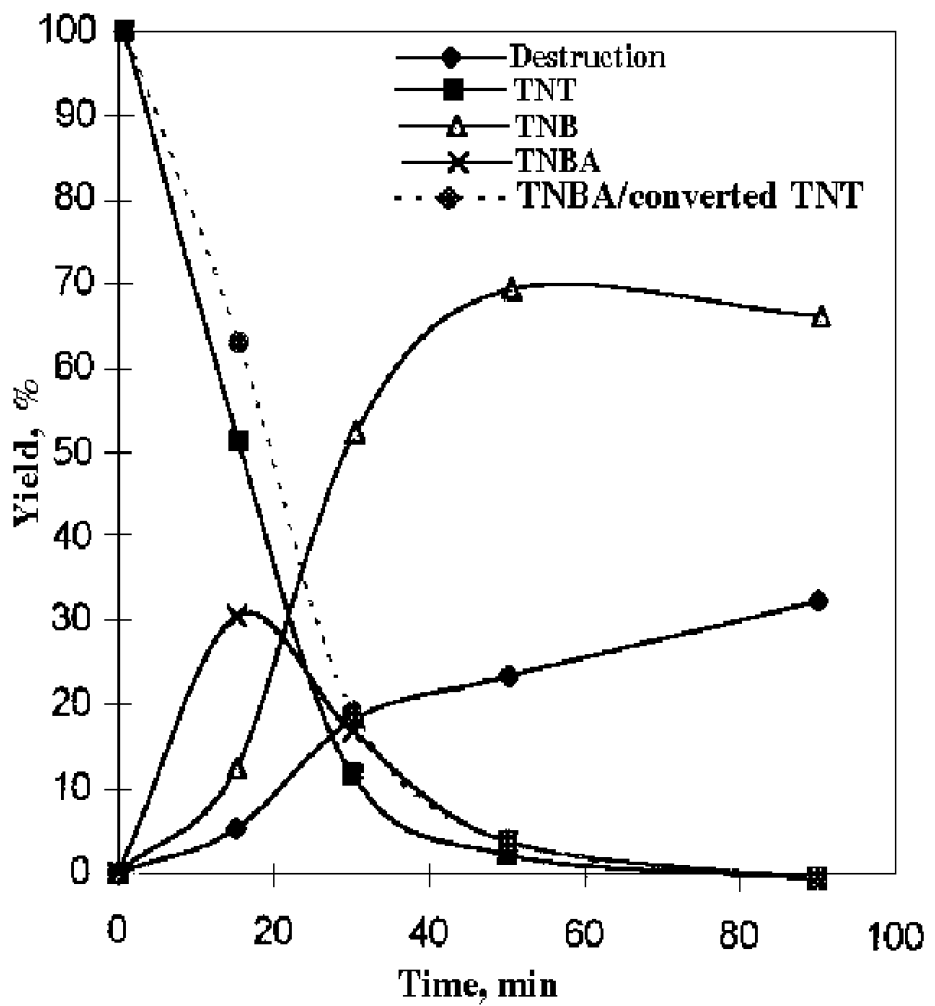

Fig. 2.1. Effect of temperature on TNT oxidation. 

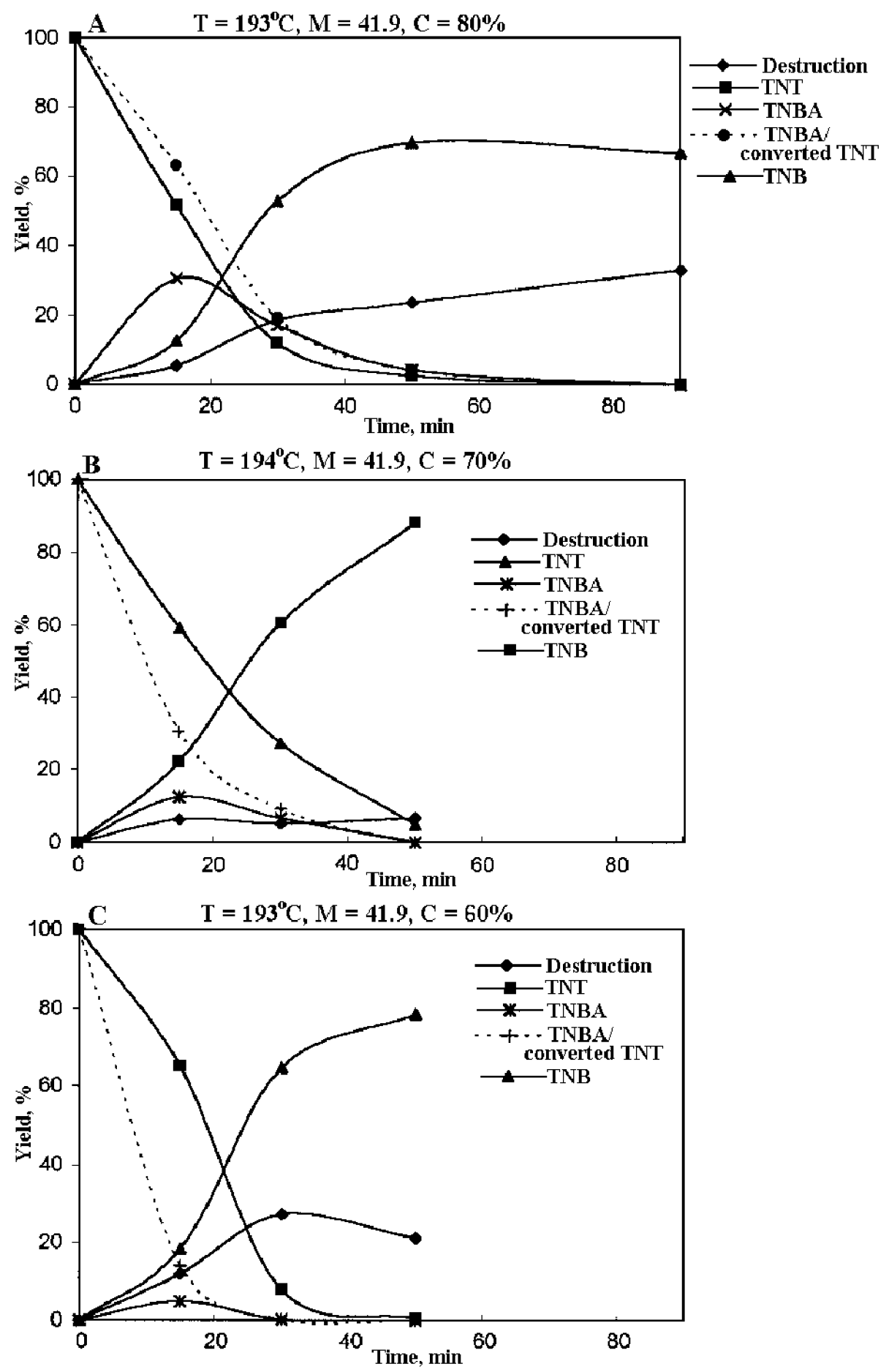

Fig. 2.2. Effect of nitric acid concentration (C) on TNT oxidation kinetics. 
We found that the accumulation of nitrogen oxides in the reaction mixture had a dramatic effect on the process by increasing significantly the TNB fraction in the reaction products. Key optimization factors are the maintenance of the maximum acidity in the course of the process, the removal of nitrogen oxides, and the optimization of the reaction time. These steps can be achieved not only by controlling the temperature and the initial ratio between the reactants, but also by affecting the liquid-gas mass-exchange processes (either by adding oxygen for partial regeneration of nitric acid during the course of the process or by varying the pressure in the system).
Experiments performed in model reactor tubes with air supply to the autoclave, and with pressure controlled by the routine removal of gases, showed that the performance characteristics of the process can be improved (an increase in the current and total yields of the target product, TNBA, and widening of the optimum contact time range). Figures 2.3 and 2.4 show typical curves for the TNBA yield in the process: The experiments performed indicated that it was possible to determine the requirements for the pilot plant to be used for testing the method for the production of TNBA.

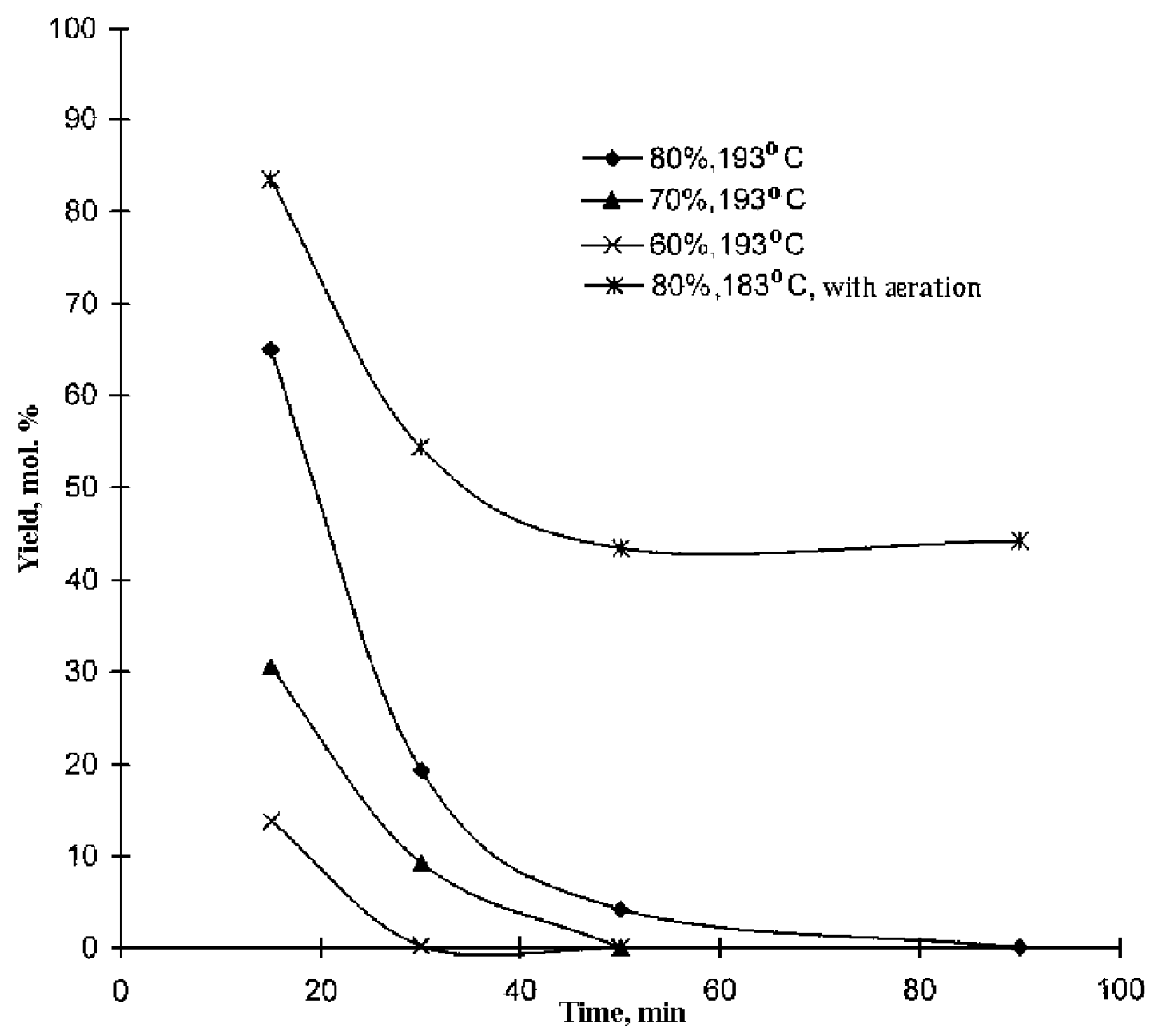

Fig. 2.3. TNT oxidation by nitric acid in autoclave. Dependence of TNBA yield (calculated on the basis of converted TNT) on oxidation conditions. 


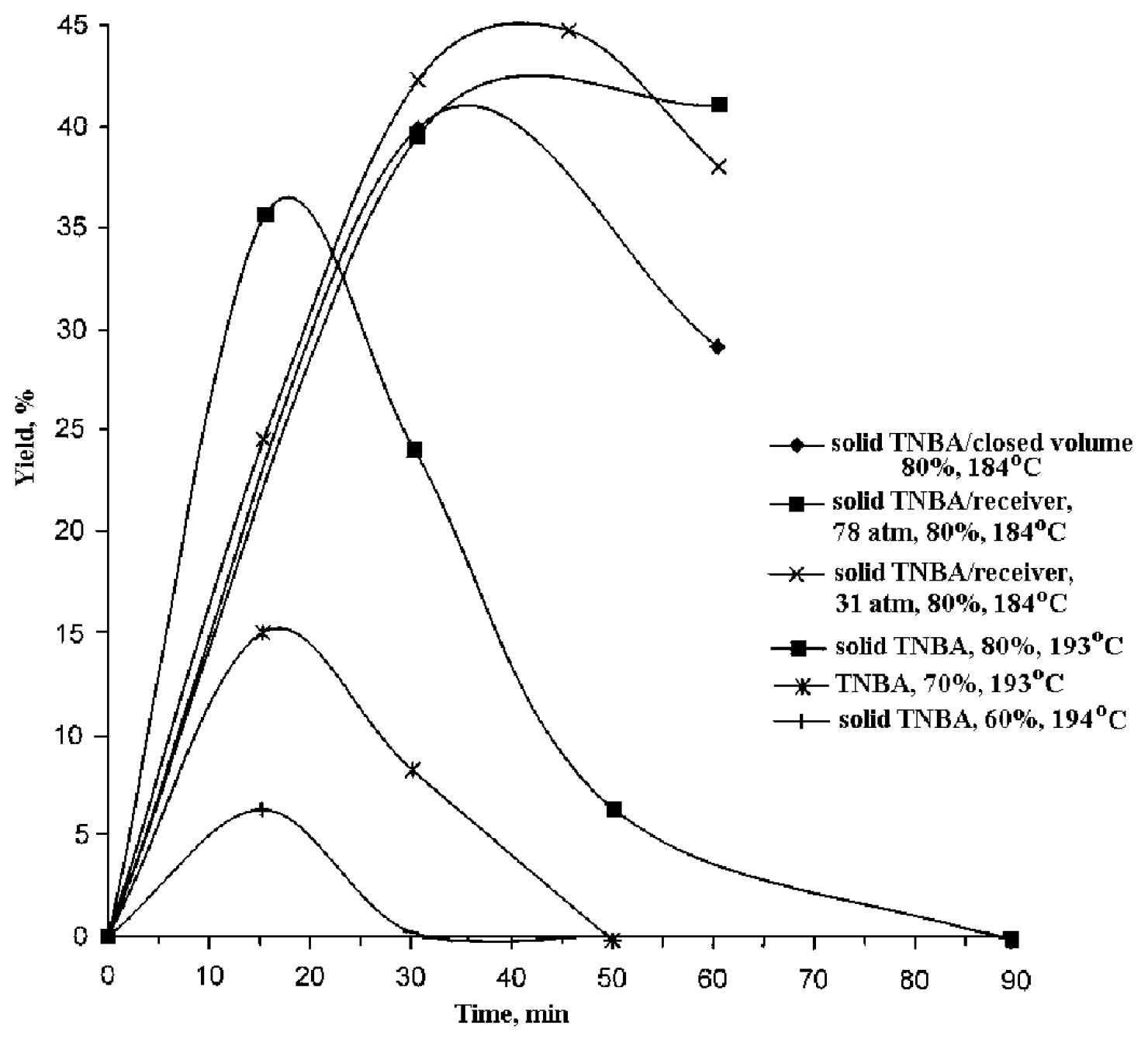

Fig. 2.4. 2,4,6-TNT oxidation by nitric acid. TNBA content in solid product $(\% \mathrm{w} / \mathrm{w})$ at different oxidation conditions. 


\section{DESIGN AND TESTING THE METHOD FOR CONTINUOUS PRODUCTION OF TNBA IN A PILOT PLANT}

Based on our studies of the kinetics and mechanisms of TNT oxidation to TNBA, a pilot plant was designed and constructed. This plant operates in the continuous mode of a reactor unit. The use of the high-temperature and highpressure reactor unit required the development of a specific apparatus and operating procedures. The products were separated in a conventional standard laboratory apparatus.

Figure 3.1 shows a schematic diagram of the pilot plant performing the following functions:

1. preparation of nitric acid in which the required concentrations of TNT are dissolved;

2. supply of the working solution at a specified flow rate into the oxidation reactor under pressure;

3. heating and monitoring the reactants at a specified temperature and pressure;

4. supply of compressed air to the reactor unit;

5. cooling of liquid and gaseous reaction products;

6. decreasing the pressure of the reaction mixture to atmospheric levels; and

7. removal of nitrogen oxides from the reaction mixture by purging with air.

The pilot plant includes:

1. A stirring apparatus for preparing nitric acid and TNT solutions. This apparatus is a $35-\mathrm{L}$ stainless steel vessel with a cap, a fixed stirrer, a thermocouple inlet, a heatexchange coil, and a loading well. The propeller agitator runs at $900 \mathrm{rpm}$. A discharge tube with a valve is located at the bottom of the vessel.

2. A pump for supplying the nitric acid and TNT solution into reactor 3 . The plunger pump has the capacity of 0 to $10 \mathrm{~L} / \mathrm{h}$, and a working pressure up to $10 \mathrm{MPa}$. All parts in contact with the working medium are stainless steel.

3. A reactor vessel for TNT oxidation. The schematic diagram of the reactor is shown in Fig. 3.2. The reactor was designed as a column apparatus of high-purity titanium with a cap attached with flanges. The total reactor volume is $6 \mathrm{~L}$. The interior of the cylinder is separated into two sections (internal and external) by a thin-walled-tube partition placed coaxially. The lower part of the partition reaches the bottom and has holes for liquid leakage from one section to the other. The upper part of the partition does not reach the top of the apparatus in order to form a gas volume common for all sections.

The temperature in the reactor is maintained by heaters at the outer walls of the reactor. The heaters automatically maintain the temperature of the outer wall of the apparatus at a specified level. A heat-distribution shield of flat copper is located between the reactor and heater walls to provide uniform heating.

Tubes for adding the starting reactants and removing the reaction products are inserted into the internal cavity of the reactor. The level of the tube for the removal of the reaction product indicates the level of liquid in the reactor. The bottom part of the internal section has a bubbler for supplying air.

4. A receiver for compressed air. A 7-L gas cylinder of stainless steel with welded tubes serving as gas inlet and outlet, and with a bottom tube for removing a condensed liquid.

5. A jacketed tube heat exchanger made of titanium with a heat-exchange surface area of $0.04 \mathrm{~m}^{2}$. 


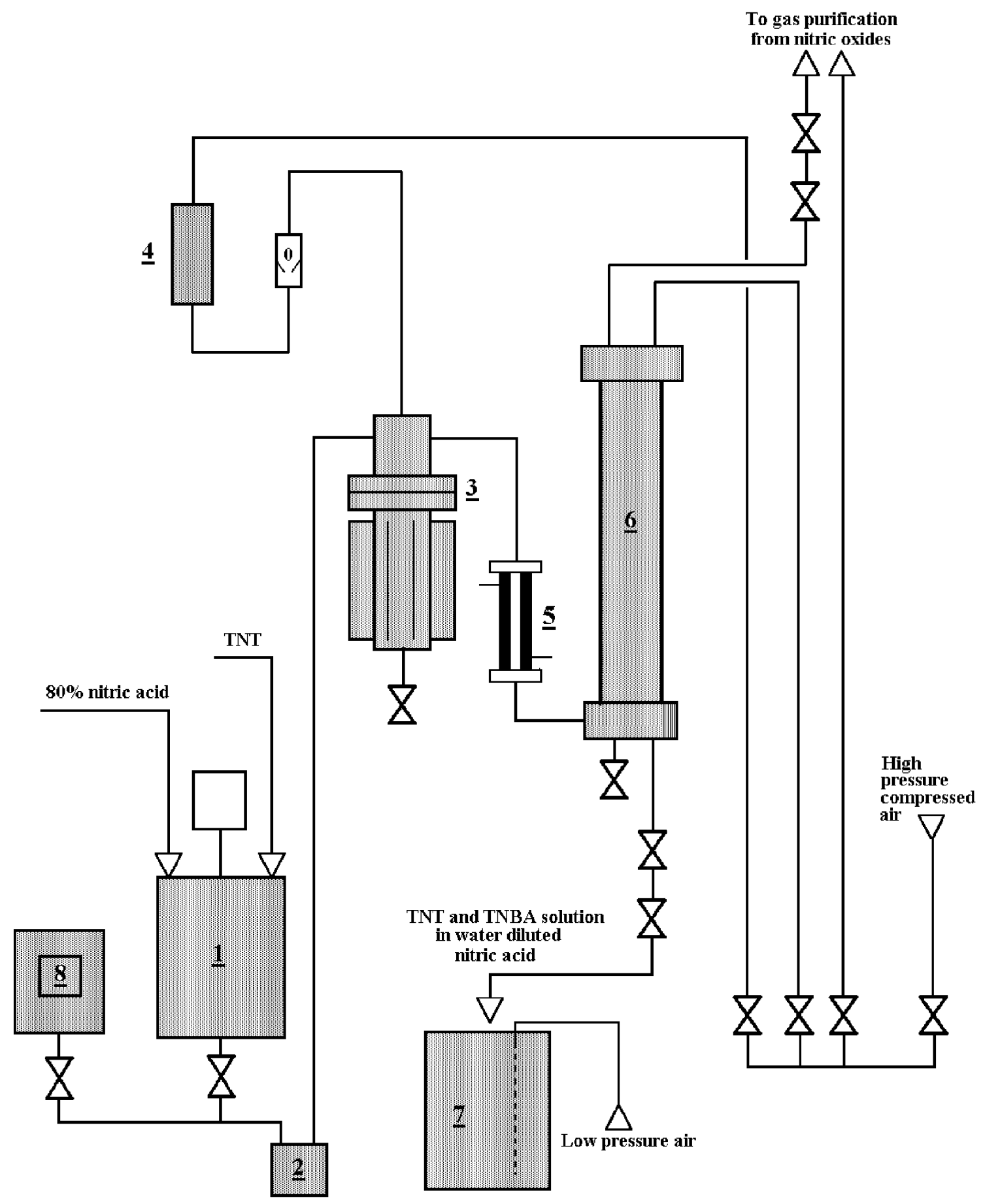

Fig. 3.1. Principal scheme of pilot plant reaction for TNBA production. 


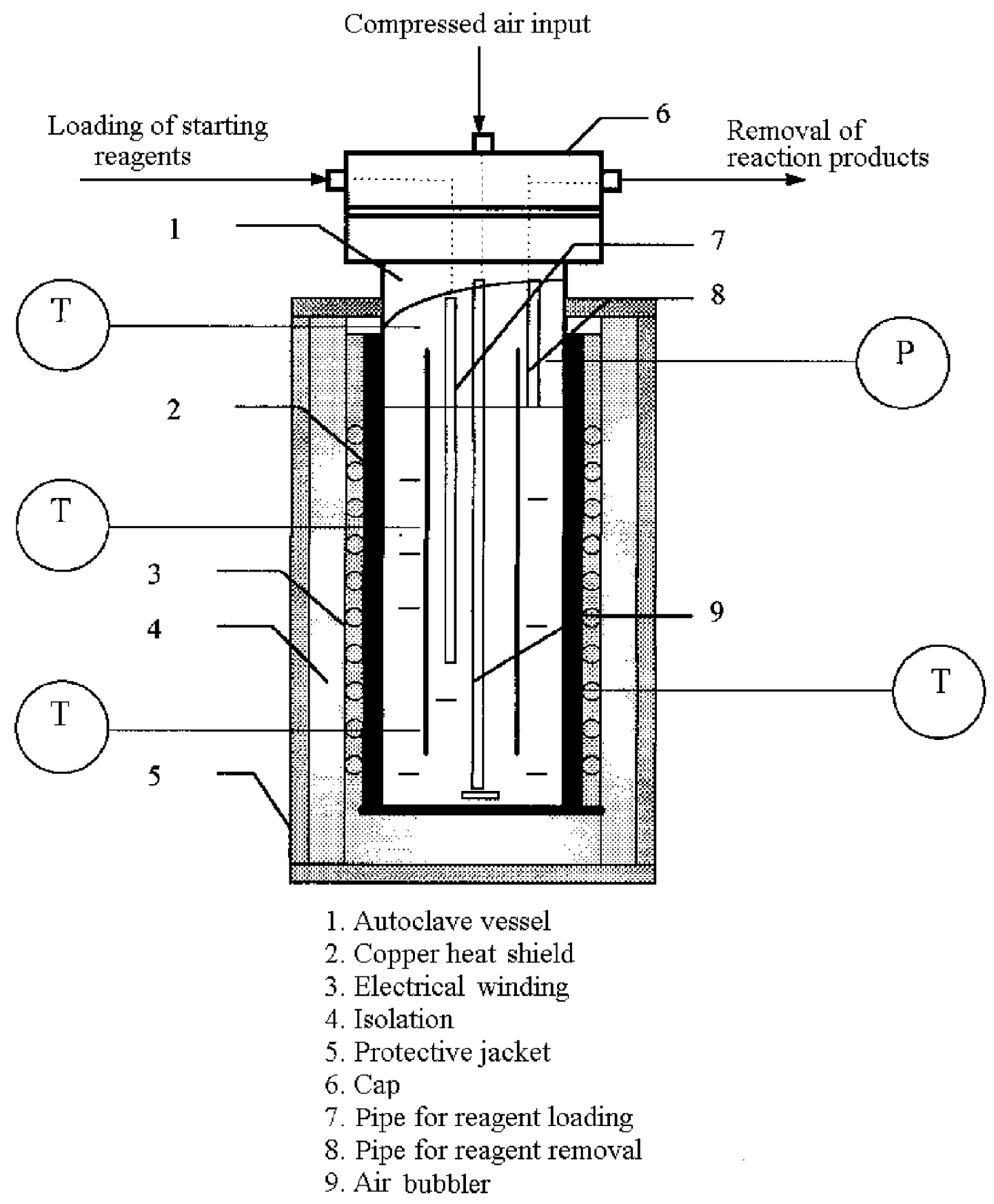

Fig. 3.2. Schematic diagram of oxidation reactor

(T-temperature sensor; P-pressure sensor). 
The cooling liquid and the reaction mixture are fed into the shell side and the tube space, respectively. The heat-exchange tubes are $8 \mathrm{~mm}$ in diameter and have a wall thickness of $1 \mathrm{~mm}$.

6. A high-pressure receiver for collection and intermediate storage of the reaction products after oxidation.

The column apparatus has a cap and a bottom attached by flanges. The apparatus volume is $12 \mathrm{~L}$; the working pressure is up to $10 \mathrm{MPa}$. A heat-exchange coil for circulating cold water during the course of the process is located at the bottom, within the apparatus.

7. A receiver for the reaction mixture after the pressure drops to atmospheric levels. This is a stainless-steel 35-L vessel with a bubbler for air supply.

8. A stainless-steel measuring tank for storage of wash acid. This unit is placed in an isolated concrete box in a separate room from the reactor vessel. The processes under pressure are remotely controlled from a control panel. The pilot plant is supplied with compressed air for control and measuring instruments, electricity, water, and ventilation from centralized systems in the laboratory building. High-pressure air is produced by a special compressor and supplied via a separate line. Vented gases are purified by passing through a filter to remove nitrogen oxides. The product was separated using standard laboratory equipment.

Trial runs of the pilot plant made it possible to develop and refine the experimental procedure and to produce batches of product to use as reference samples and for solving problems of TNBA separation from the reaction mixture.

The trial runs demonstrated that the results obtained in our studies of the kinetics of TNT oxidation can be used to select operating conditions for the reactor unit and, equally as important, that the method can be technically implemented. Thus, the pilot plant can serve as a prototype for an industrial module.

The run was performed using an initial nitric acid concentration of $80 \%$. To compare the results of kinetic studies and the pilot plant runs, all experiments were conducted using an excess of nitric acid that helped to maintain uniform temperature conditions and eliminate undesirable product precipitation in transient regimes.

The oxidation of TNT was performed in a continuous operating mode, using a homogeneous liquid reaction mixture. We thought that the kinetics of oxidation under these conditions would be similar to the kinetics of oxidation we observed in a batch autoclave.

Differences can be due to different conditions of gas exchange and an ambiguity in the term 'residence time' as applied to a reactor vessel as opposed to a flow unit. To compensate for these factors, experiments with purging air, in which a reasonably wide range of contact times in the reactor vessel was observed, were taken as a basis.

The temperature of the reaction mixture in a steady-state regime was maintained at a level of 180 to $190^{\circ} \mathrm{C}$. An $80 \%$ nitric acid solution was used for oxidation. The volume of the continuous solution supplied was chosen from a calculated residence time in the reactor of 25 to $30 \mathrm{~min}$. This calculation was performed using the ratio between the flow rate of the initial solution and the reactor volume filled with the liquid. The actual residence time of microvolumes in the reactor will be somewhat shorter because it also includes the time to heat the mixture. Moreover, the residence times for particular microvolumes of the flow will be somewhat different.

The pressure was maintained at a level of 3.5 to 4.5 MPa to provide a liquid-phase balance and to ensure minimum solubility of nitrogen oxides. 
Compressed air was supplied continuously to the reactor through the bubbler during the entire process to decrease the amount of dissolved nitrogen oxides in the liquid phase.

The TNT oxidation in the pilot plant was performed typically as follows:

An $80 \%$ nitric acid solution was prepared in agitator apparatus 1 by mixing calculated amounts of $98 \%$ nitric acid and water. A portion of the nitric acid solution obtained was placed in wash acid measuring tank 8 . TNT was added in portions to the $80 \%$ nitric acid in agitator apparatus 1, and the mixture was stirred until the TNT was completely dissolved. The nitric acid solution from wash acid measuring tank 8 was pumped into the reactor unit using a measuring pump until reactor 3 was full.

A pressure of 3.0 MPa was produced in the reactor unit (reactor 3, heat exchanger 5, and a high-pressure receiver 6 interconnected by tube lines) using compressed air from a high-pressure compressor (not shown). The system was tested for leaks by the absence of a pressure drop over a given time interval. Operation of liquid and gas valves was tested by check actuation from the panel, then heaters of the reactor were turned on, and the temperature within the apparatus was increased to between 180 and $190^{\circ} \mathrm{C}$.

Simultaneously, cold water was supplied to heat exchanger 5.

The reactor temperature was automatically maintained at a specified level by resistor heaters located in the outer walls of the reactor. The pressure was automatically maintained at a specified level using unloading valves installed on high-pressure receiver 6 . When the temperature in reactor 3 attained a steady-state value, TNT supply to the reactor was turned on.

The TNT solution was fed continuously via the inlet tube to the inner section of the reactor under the liquid layer, where it was heated by heat exchange with the heated reaction mixture and the heat released in the oxidation process.
The liquid flowed from the inlet point to the bottom of the vessel. Gases released in the oxidation process moved as a countercurrent with respect to the liquid flow and provided adequate mixing of the reaction mixture. The major portion of gaseous products was released within the inner section. The inner section is similar to a mixing reactor with respect to heatand mass-exchange processes. Next, the direction of the liquid flow was reversed, and the liquid flowed from the bottom to the top. The character of motion is close to that of a plug-flow reactor because of the low speed of the liquid.

Different hydrodynamic regimes for the initial and final stages of the chemical process can occur within a single apparatus because of reactor sectioning.

Air was supplied to the reactor bubbler 20 to $30 \mathrm{~min}$ after the addition of TNT began. The pressure in receiver 4 (which was 0.2 to 0.5 MPa higher than the pressure in the reactor unit) was produced using a compressor. Air entered the reactor bubbler from receiver 4 through a back valve and a throttling sleeve. The air flow rate was controlled using the throttling sleeve by changing the pressure difference between the receiver and the reactor unit. The air flow rate was maintained at 100 to $300 \mathrm{~mL} / \mathrm{min}$ throughout the process.

Gaseous and liquid products were removed from the reactor via the upper fitting. The bottom level was adjusted so that the liquid-phase volume was $3 \mathrm{~L}$ (a coefficient of the reactor charge of 0.5 ). The required residence time of the reaction mixture was controlled by varying the flow rate of the TNT solution, which was usually 6 to $8 \mathrm{~L} / \mathrm{h}$.

After leaving reactor 3, liquid and gaseous reaction products were cooled with water to 80 to $100^{\circ} \mathrm{C}$ in heat-exchanger 5 and collected in high-pressure receiver 6 . The high-pressure receiver was a titanium column with a cooling coil in the bottom. The receiver served as an intermediate accumulator of the reaction 
mixture and as a buffer apparatus for smoothing off pressure pulsation of discharge facilities and protection systems.

In a steady-state mode of operation, the system was discharged via the bottom throttling ball valve operating in an automatic mode with respect to the pressure in the system.

The upper valve acted only upon increasing the pressure above operating valves and served as an emergency valve. Additionally, the pressure can be released manually using special facilities.

After decreasing the pressure to atmospheric pressure, the reaction mixture was collected in an open vessel placed under the hood. Air was supplied continuously to the vessel through a bubbler to remove dissolved nitrogen oxides. The nitrogen oxides released from the solution were vented and then trapped in a special system.

The reaction mixture was further processed in a standard apparatus under atmospheric pressure. It was evaporated under vacuum produced by a water-jet air pump until the organic phase content reached 25 to $30 \%$, and then it was diluted with 2 volumes of water. The precipitate formed was filtered off.

TNBA was extracted from the precipitate by dissolving in an aqueous $\mathrm{NaHCO}_{3}$ solution at room temperature. The dissolution was performed by adding the mixture in portions to the prepared $\mathrm{NaHCO}_{3}$ solution with intense stirring to provide gas evolution without foam formation. After filtration of the solution from the undissolved residue, the filtrate was acidified with nitric acid to separate TNBA. The acidification was performed in an agitator apparatus by adding the TNBA salt to an aqueous nitric acid solution. The precipitate was filtered off, washed with cold water on the filter, and dried under mild conditions in a direct-fired drier in an air flow at 30 to $40^{\circ} \mathrm{C}$ until a constant weight. The product obtained contained no less than $98 \%$ TNBA. (If necessary, the product of higher purity can be obtained by recrystallization from nitric acid.)

The precipitate, undissolved in the $\mathrm{NaHCO}_{3}$ solution, is a mixture of TNT and TNB. The ratios among TNBA, TNT, and TNB depend on the oxidation conditions. On these experiments, TNBA was prepared under conditions where TNT was present almost exclusively in the undissolved precipitate in the $\mathrm{NaHCO}_{3}$ solution. TNB impurities constituted no more than 2 to $3 \%$; thus, we concluded that this product can be used for repeated oxidation.

In this case, the TNT conversion was 25 to $30 \%$, and the TNBA yield was about 65 to $70 \%$ on the basis of converted TNT. This value is consistent with the values expected based on our kinetic curves at the given conversion.

We believe that the yield can be increased; however, the process should be optimized further, primarily with respect to the residence time, temperature, pressure, and air flow rate. Moreover, because this is a continuous process, the possibilities for controlling and modifying it are considerable.

In general, the experiments demonstrate that the process can be implemented on an industrial scale, and that the approaches to the design of such implementation are straightforward. Of course, the development of a particular process will require extensive studies on the optimization of particular parameters and stages and the development of a balanced massexchange system. However, the data obtained make it possible to decide on the implementation of this method in industry. 


\section{OXIDATION OF TNT TO TNBA ON AN INDUSTRIAL SCALE}

Our experiments have shown that TNBA can be produced in acceptably high yields by TNT oxidation with nitric acid under mild conditions. However, a key feature of the process is that, under any conditions, TNBA occurs in a mixture along with TNT and TNB. The amounts of TNT and TNB are determined by the incomplete conversion of TNT to TNBA and the occurrence of the secondary reaction of TNBA decarboxylation, respectively. Thus, the process of TNBA production must involve separation of the target product, TNBA, and the utilization of TNT and TNB obtained in the course of the separation. Two approaches to implement the process and to determine the conditions for TNT oxidation are possible:

1. If TNBA is to the sole target product of the process, oxidation should be performed at a low TNT conversion level (20 to 30\%). In this case, the primary objection is to minimize TNB impurities. The TNT separated from TNBA can be used repeatedly for oxidation. Despite the fact that the TNBA yield is not maximum under these oxidation conditions, the total yield on the basis of the amount of starting material will be reasonably high. However, large amounts of reactants will need to be recycled.

2. If TNB is to be produced in addition to TNBA, the possibilities for optimizing the process are significantly enhanced. A maximum TNBA concentration in the organic product after oxidation should be the main criterion for the optimization of the oxidation process. In this case, a significantly larger per-pass TNBA amount can be obtained in the reactor.

Nonacid organic products (a mixture of TNT and TNB) can be converted into TNB by repeated oxidation under more severe conditions. This mode of operation will exhibit better performance characteristics because recycling is not required, and the mass transfer per unit mass of the commercial product will be decreased. The thermal oxidation method of TNB production by TNT oxidation was developed at the SKTB "TEKHNOLOG" and introduced into industry. However, because of severe conditions of oxidation, the setup is more sophisticated.

From the standpoint of TNBA preparation and separation, both of the above processes are similar, and the differences concern only the processing of nonacid organic impurities separated from the target product. The industrial process that we suggest is illustrated in Fig. 4.1.

Oxidation is performed with a large excess of $80 \%$ nitric acid (a 9 to $10 \%$ TNT solution) in a continuous operation. The working solution supplied for oxidation is prepared at regular intervals to coincide with the continuous operation of the oxidation unit. The working solution is prepared from fresh $98 \%$ nitric acid, distilled 75 to $90 \%$ nitric acid, and fresh or recycled TNT. For dilution, 35 to $45 \%$ acid from absorption units can be used. (A large excess of nitric acid provides stable oxidation and is favorable for safety conditions.)

After oxidation, the pressure of the reaction products is decreased to atmospheric levels. Nitrogen oxides are removed and trapped. Nitrogen oxide waste gases can be purified by conventional industrial procedures. The nitric acid obtained after absorption with water can be used for diluting concentrated nitric acid in the first stage of the process.

The reaction mixture is then evaporated under vacuum until the residual concentration of the organic product reaches 25 to $40 \%$. The distillate is recycled to the TNT preparation stage. In this case, 60 to $70 \%$ of nitric acid is recycled to the oxidation stage. A portion of nitric acid is used for acidification of an alkaline TNBA solution at the stage of separation of the target product. 


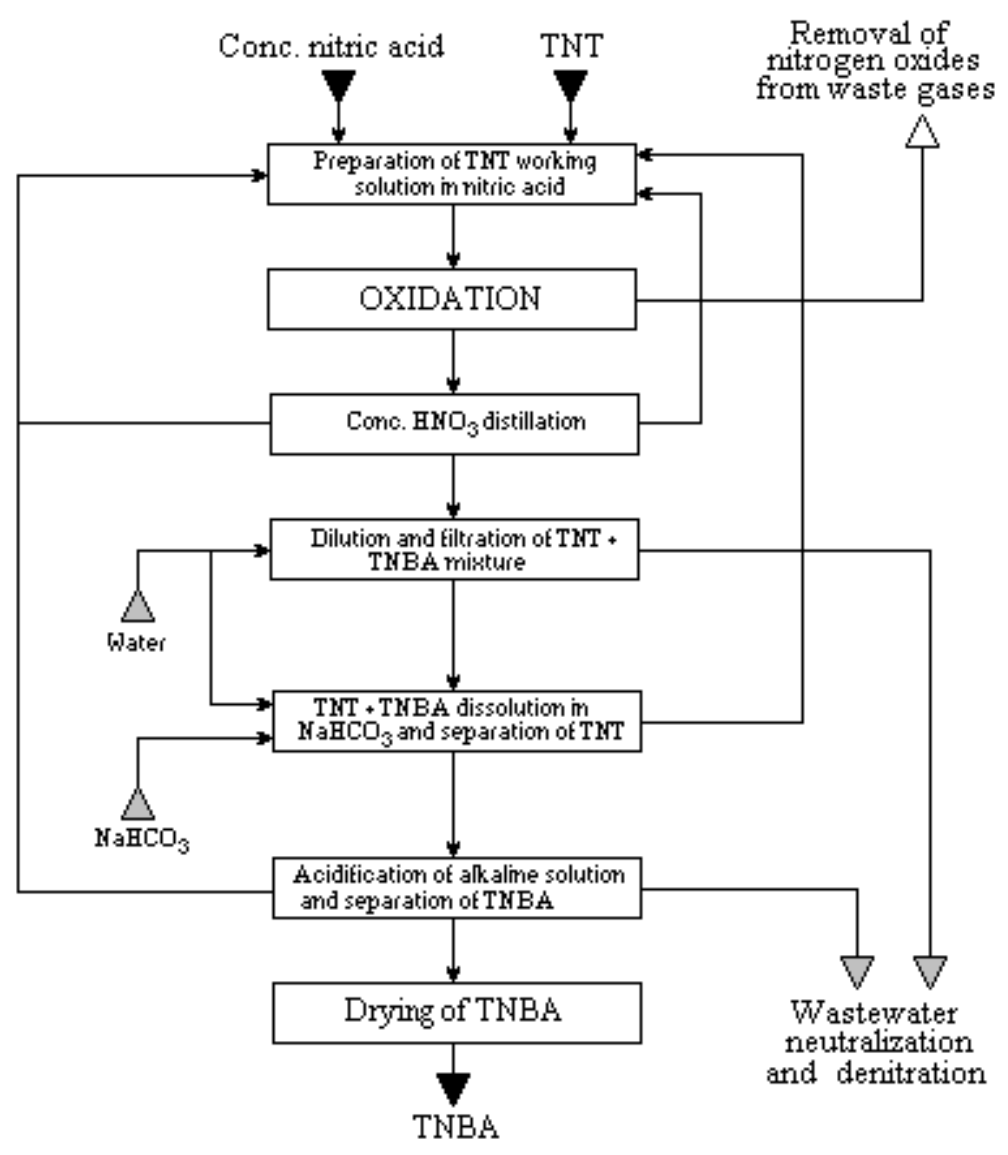

Fig. 4.1. Flowchart illustrating TNBA production by TNT oxidation with nitric acid, including recycling of TNT.

The bottoms are diluted with 2 to 3 vol \% of water, stirred, and the formed solid precipitate containing TNT and TNBA with TNB impurities is filtered. The mother liquors are either incinerated or purified by adsorption.

TNBA is extracted from the filtered product by conversion into a water-soluble salt. For our purposes, the product is dissolved in a sodium bicarbonate solution at room temperature. The undissolved residue is primarily TNT with trace amounts of TNB. TNBA is separated by filtration and recycled to the oxidation stage.

The 10 to $15 \%$ solution of the sodium salt of TNBA is acidified by distilled nitric acid, and the precipitate is filtered off, washed with cold water on the filter, and dried in direct-fired dryers in an air flow at a temperature no higher than $45^{\circ} \mathrm{C}$.

Nitrogen oxide waste gases can be purified in accordance with sanitary regulations by conventional industrial procedures.

Nitric acid that cannot be recycled and mother liquors should be neutralized. Nitro products contained in wastewater are efficiently extracted by sorption on a charcoal followed by incineration; this is a commonly used industrial procedure.

TNBA can also be separated without reprecipitation using the differences in the solubilities of TNBA, TNT, and TNB. However, this method is more suitable for the second version, which includes the conversion of the 
organic product separated from TNBA into TNB.

Thus, we have shown that the production of TNBA by TNT oxidation is reasonably sophisticated but a technically feasible process. The commercial product obtained is adequately pure for the majority of applications. If necessary, TNBA can be further purified by recrystallization or reprecipitation from nitric acid. Spent nitric acid can be recycled.

The process can be further defined after the development of optimization criteria and the determination of technical and economic limitations.
For now, the main goal in the refinement of the process should be the development of a balanced system of recycling flows and the optimization of oxidation conditions. All of these works can be performed using the pilot plants and the experience of the SKTB "TEKHNOLOG" in the development of thermal oxidation processes in aqueous nitric acid media. 


\section{CONCLUSIONS}

The production of 2,4,6-trinitrobenzoic acid (TNBA) by the oxidation of 2,4,6-trinitrotoluene (TNT) with aqueous nitric acid solutions under pressure in specially designed reactor vessels was examined. We found that the TNT

oxidation at 180 to $190^{\circ} \mathrm{C}$ in 60 to $80 \%$ nitric acid resulted in three products: $1,3,5-$ trinitrobenzene (TNB), TNBA, and products of complete decomposition. The TNBA yield is time-dependent and is related to the amount of TNT converted. We found that TNBA can be produced with a maximum yield of $80 \%$ (based of reacted TNT). The best results were obtained during oxidation with an excess of $80 \%$ nitric acid at 180 to $190^{\circ} \mathrm{C}$, with a supply of air to the reactor vessel at a pressure maintained at the lowest limit that maintains the liquid-phase process.
The methodology for a continuous process was developed and operated in a specially designed pilot plant. We demonstrated that the key technical problems can be solved; the results of kinetic studies can be confirmed in the pilot plant; and the methods for separating the target product from the reaction mixture were effective.

Pilot batches of TNBA of adequate quality were produced, and the samples were delivered to the customer.

A schematic of an industrial process for the production of TNBA as an individual target product or for the combined production of TNB and TNBA is proposed. 


\section{REFERENCES}

1. S. G. Zlotin, E. A. Serebryakov, P. G. Kislitsin, L. D. Konyushkin, V. V. Semenov, A. C. Buchanan III, and A. A. Gakh, "Nitro1,2-Benzoisothiazoles and Isothiazolones from Polynitroaromatic Compounds and Related Chemistry," Abstract Paper, The ACS National Meeting, New Orleans, 218: 48-Orgn., Part 2, Aug. 22, 1999.

2. S. G. Zlotin, P. G. Kislitsin, A. I. Gerasyuto, L. D. Konyushkin, "Reactions of 4,6-Dinitro1,2-Benzoisothiazoles and Isothiazolones with N-Nucleophiles," Abstract Paper, The ACS National Meeting, New Orleans, 218: 60-Orgn., Part 2, Aug. 22, 1999.

3. S. G. Zlotin, P. G. Kislitsin, A. V. Samet, E. A. Serebryakov, L. D. Konyushkin, V. V. Semenov, A. C. Buchanan, III, A. A. Gakh, "Synthetic Utilization of Polynitroaromatic Compounds, I. S-Derivatization of 1Substituted 2,4,6-trinitrobenzenes with Thiols," J. Org. Chem., 2000, in press.
4. S. G. Zlotin, P. G. Kislitsin, A. I. Podgursky, A. V. Samet, V. V. Semenov, A. C. Buchanan III, and A. A. Gakh, "Synthetic Utilization of Polynitroaromatic Compounds. II. Synthesis of 4,6-Dinitro-1,2Benzisothiazol-3-Ones and 4,6-Dinitro-1,2Benzisothiazoles from 2-Benzylthio-4,6Dinitrobenzamides, " J. Org. Chem., 2000, in press.

5. Syntezy Org. Preparatov (Russ), 1949, v.1, 416 (Organic Synthesis, 1941, v. 1; D. R. P. 127,325; Frdl. 1901, 6, 148).

6. E. Bengtsson, N. Billingsson, K.-J. Persson, B.-O. Backman, "Method of Producing Symmetrical Trinitrobenzene," U.S. Patent No. 4334110; June 8, 1982; CA 095 (03) $024518 \mathrm{~N}$. 


\section{INTERNAL DISTRIBUTION}
1. A. C. Buchanan III
9. C. H. Shappert
2-6. A. A. Gakh
10. Laboratory Records - RC
7. M. R. Guerin
11-12. Laboratory Records for Submission to OSTI
8. M. L. Poutsma
13. Central Research Library

\section{EXTERNAL DISTRIBUTION}

14-20. A. A. Astrat'ev, St. Petersburg Institute of Technology, SKTB “Technolog” Sovetsky Pr., 33a, St. Petersburg, 193117, Russia

21. J. Noble, U.S. Department of Energy, NN-41/IPP, 1000 Independence Avenue, SW, Washington, D.C. 20585

22. P. Green, U.S. Department of Energy, NN-41/IPP, 1000 Independence Avenue, SW, Washington, D.C. 20585

23. Office of the Assistant Manager for Energy Research and Development, DOE Oak Ridge Operations (DOE-ORO), P.O. Box 2008, Oak Ridge, TN 37831

24. J. Rooney, U.S. Department of Energy, NN-41, 1000 Independence Avenue, SW, Washington, D.C. 20585

25. V. V. Semenov, Zelinsky Institute of Organic Chemistry, Leninsky Pr., 47, Moscow, 117913, Russia

26. C. K. Williams, MS 8257, P. O. Box 2009, Oak Ridge, TN 37831 